Research Paper

\title{
ApoM is an important potential protective factor in the pathogenesis of primary liver cancer
}

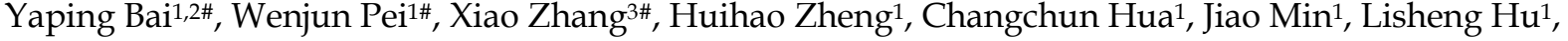 \\ Shuangqiu Du ${ }^{1,2}$, Zuyue Gong1, Jialin Gao ${ }^{1,4}$, Yao Zhang ${ }^{1,2}$ \\ 1. Anhui Province Key Laboratory of Biological Macro-molecules Research, Wannan Medical College, Wuhu 241002, China \\ 2. Department of Biochemistry and Molecular Biology, Wannan Medical Collage, Wuhu 241002, China. \\ 3. Department of Pediatric Surgery, The First Affiliated Hospital Of USTC-Division of Life sciences and medicine, University of China, Hefei 230001, China. \\ 4. Department of endocrine, The First Affiliated Hospital of Wannan Medical College, Wuhu 241002, China. \\ \#These authors contributed equally to this work. \\ 凶 Corresponding author: Yao Zhang, E-mail: zhang18905537001@163.com; and, zhangyao@ahedu.gov.cn.
}

(1) The author(s). This is an open access article distributed under the terms of the Creative Commons Attribution License (https://creativecommons.org/licenses/by/4.0/). See http://ivyspring.com/terms for full terms and conditions.

Received: 2020.09.10; Accepted: 2021.04.24; Published: 2021.06.04

\begin{abstract}
In recent years, abnormal liver lipid metabolism has emerged as one of the important pathogenesis pathways of primary liver cancer. It is highly important to identify the mechanisms to explore potential prevention and treatment targets. Apolipoprotein $M$ is specifically expressed in the liver and participates in liver lipid metabolism, but the evidence that ApoM affects primary liver cancer is insufficient. The Cancer Genome Atlas (TCGA) database and clinical case analysis, as well as animal level and cell level analysis suggest that the expression level of ApoM gene in cancer tissues is lower than that in paracarcinoma tissues. Further experimental research found that the deletion of ApoM significantly increased the proliferation of mouse liver cancer cells (Hepa 1-6) and inhibited the level of apoptosis induced by cisplatin. In addition, mouse liver cancer cells lacking ApoM showed stronger migration and invasion capabilities in transwell experiments. In contrast, overexpression of ApoM in Hepa 1-6 cells and Huh-7 cells showed an inhibition of proliferation, up-regulation apoptosis and reduced migration and invasion. In vivo, the deletion of the ApoM accelerated tumorigenesis in nude mice and allowed the mice to develop liver tumor mutations more quickly under the induction of $\mathrm{N}$-nitrosodiethylamine and the survival time of mice was shorter than that control. Therefore, ApoM may be a potential protective factor to inhibit the occurrence and development of primary liver cancer.
\end{abstract}

Key words: apolipoprotein M; protective factor; primary liver cancer

\section{Introduction}

Primary liver cancer is one of the most common malignant tumors, with a high mortality rate worldwide, and its incidence is increasing year by year [1]. In view of its high degree of malignancy and often undetected development, most patients have entered the middle and advanced stages when diagnosed. Therefore, the study of the pathogenesis of primary liver cancer is a major scientific issue that needs to be resolved urgently. Previous studies have shown that the alcoholism, smoking, chronic viral hepatitis, liver cirrhosis, and aflatoxin are risk factors for liver cancer [2]. However, not all patients who present with primary liver cancer have these risk factors, and so it is possible that some other important factors have been ignored. Metabolic disorders, especially lipid metabolism disorders, are an important microenvironment for tumor pathogenesis. At present, the "metabolic disease" theory is being applied in relation to the onset of primary liver cancer $[3,4]$. Changes in liver lipids metabolism are closely related to the occurrence of liver cancer, and nonalcoholic fatty liver disease may be identified as one of the main causes of primary liver cancer in the future [5].

As a member of the apolipoprotein family, Apolipoprotein $\mathrm{M}$ (ApoM) has been shown to be involved in related processes of lipid metabolism. Research on ApoM has mostly focused on atherosclerosis. For example, the HDL-ApoM-S1P complex can protect the vascular endothelium and has a certain anti-atherosclerotic activity $[6,7]$. In addition, our previous studies have shown that 
autophagy dysfunction caused by ApoM deficiency is an important factor in liver steatosis [8]. Is it now appropriate to think about the relationship between ApoM and liver cancer? Other studies have shown that miRNA-573, which directly targets the negative regulation of $A p o M$, can promote tumor cell proliferation by inhibiting the apoptosis of liver cancer cells in vitro [9]. However, it is worth noting that the development of liver cancer in the body is a long and complicated process, during which changes in the environment in the body cannot be ignored.

There are many factors that affect the formation and development of tumors, mainly changes in the levels of tumor cell proliferation, apoptosis, migration, and invasion caused by changes in the physical and chemical environment of the body [10, 11]. Tumor cells have unlimited ability to proliferate and divide. This abnormal proliferation may be closely related to the disorder of the tumor cell cycle, which is a typical biological feature of cancer [12, 13]. Apoptosis is a method of programmed cell death regulated by genes, and it is an essential function the body uses to maintain normal physiological homeostasis [14]. In malignant tumor cells, the apoptosis process is blocked and the cell cycle regulation mechanism is destroyed, so that the rapid and massive proliferation of tumor cells is characteristic of cancer. To date, many postoperative chemotherapy drugs for cancer have been developed based on the apoptosis mechanism [15, 16]. The migration and invasion capabilities of tumor cells also determine the outcome of the disease, and which induced metastasis really threatens patients and troubles doctors $[17,18]$.

Considering all of these factors, it is reasonable to suspect a connection between ApoM and primary liver cancer. Therefore, it is important to analyze whether the ApoM gene is involved in the occurrence and development of liver cancer. This study systematically explored the role of ApoM in the growth of primary liver cancer cells including the aspects of proliferation, apoptosis, migration, and invasion. In addition, the influences of the ApoM gene on the initiation of liver cancer were aslo examined by observing the rate of tumor formation in vivo model induced by N-nitrosodiethylamine. This work will add a new basis to the theory of the correlation between apolipoprotein and liver tumors, and further enrich functional research on apolipoprotein.

\section{Materials and methods}

\section{TCGA database screening}

Software: R (version 3.6.3) (statistical analysis and visualization); $\mathrm{R}$ package: mainly ggplot2 (for visualization); Statistical method: Use paired sample T test; Molecule: APOM [ENSG00000204444]; Data: TCGA (https://portal.gdc.cancer.gov/) LIHC (hepatocellular carcinoma) project RNAseq data in level 3 HTSeq-FPKM format; Data filtering: keep paired samples; Data conversion: RNAseq data in FPKM (Fregments Per Kilobase per Million) format is converted to $\log 2$ and then compared between samples; Significance mark: ns, $p \geq 0.05 ;{ }^{*} p<0.05$; ${ }^{* *} p<0.01 ;{ }^{* * *} p<0.001$.

\section{Clinical pathology sample}

The clinical cases of liver cancer collected in the study were all from the Pathology Bank of the First Affiliated Hospital of Wannan Medical College, and all pathological samples were subject to the informed consent of the patients.

\section{Cell culture}

Primary liver cancer cells Hepa1-6 and Huh-7 (Pronox, Wuhan) are in high-glycemic DMEM (GIBCO, USA) containing $10 \%$ fetal bovine serum (ExCell, AUS) and 1\% P/S (GIBCO, USA). Mouse normal hepatocyte AML12 (ATCC, United States) cells are contained in 10\% fetal bovine serum (ExCell, AUS), 1\% Insulin-transferrin-sodium (Sigma, USA) and $0.02 \%$ dexamethasone (Sigma, USA) RPMI-1640 culture solution (GIBCO, USA), and incubate at $37^{\circ} \mathrm{C}$ in an environment with $5 \% \mathrm{CO}_{2}$.

\section{Immunohistochemistry (IHC) staining}

Twenty-three clinical liver cancer samples were collected from the pathology bank of Yijishan Hospital, Wannan Medical College, for paraffin sectioning. After antigen recovery and staining, ImageJ was used to count the average density (AOD) of ApoM in cancerous tissues and adjacent tissues, and pathological analysis of 23 clinical specimens was performed to perform statistics on gender, age, presence or absence of masses, liver cirrhosis, and necrosis.

\section{Crispr/Cas9 vector construction and infection}

The ApoM gene deletion model of Hepa1-6 and Huh-7 cells was obtained by designing sgRNA sequence, packaging and infecting lentivirus. This sgRNA sequence: Hepa1-6-ApoM-/-F: CACCGAAAA GTTGCCAACTCTTCCG, R: AAACCGGAAGAGTT GGCAACTTTTC; Huh-7-ApoM-/-F: CACCGTCAGG TGGTAGATCCATTTC, R: AAACGAAATGGATCTA CCACCAGGC were designed and obtained through the online website ccTop.com and connected to the Lenticrispr-v2, Lenticrispr-dual-puro vector. The CDS region of ApoM was amplified and ligated into the PECMV-3xflag-c vector to package the lentivirus to obtain an overexpression model of the Hepa1-6-ApoM 
gene. The primers were: Hepa1-6-ApoM-OP-F: CGGG ATCCCCACCATGTTCCACCAAGTTTGGGCAG, R: CCCTCGAGCTTGCTGGACAGCGGGCAGGCCT.

\section{Edu experiment}

Edu kit was purchased from Guangzhou Ruibo (C10310-1). Each group of logarithmic growth phase cells was taken, reagent A was added, incubated for 2 hours, fixed with $4 \%$ paraformaldehyde, incubated with $2 \mathrm{mg} / \mathrm{mL}$ glycine for 5 minutes, washed with PBS, added $0.5 \%$ TritonX-100 PBS penetrant, incubated for 10 minutes, and washed with PBS once. Reagent $\mathrm{B}$ was added and incubated for 30 minutes; washed with penetrant twice, incubated with $1 \mathrm{X}$ Hoechst 33342 reaction solution for $0.5 \mathrm{~h}$, washed with PBS, and observed and analyzed with fluorescence inverted microscope (Olympus, Japan) $10 \times 10$.

\section{Cell cycle detection}

Cells were collected according to the instructions of the cell cycle detection kit (China KGI, Cat: KGA105-KGA108), washed with PBS (2000 rpm, 5 $\mathrm{min})$, prepared in a single cell suspension, centrifuged, and removed from the supernatant; 70\% ice ethanol was added to the cells and fixed overnight at $4{ }^{\circ} \mathrm{C}$. The supernatant was discarded after centrifugation, 1:9Rnase A:PI staining working solution was added and incubated for $30 \mathrm{~min}$ at room temperature in the dark. Flow cytometer (BD, USA) was used to detect.

\section{Flow cytometry}

Cells were collected according to the instructions of the Apoptosis Detection Kit (BD, USA, Cat: 556547), then resuspended in pre-cooled $1 \times \mathrm{PBS}$ and washed twice. $300 \mu \mathrm{L}$ of $1 \times$ Binding buffer was added to resuspended cells, and then $5 \mu \mathrm{L}$ of AnnexinV-FITC to mix well (BD, USA) was added and incubated at room temperature in the dark for 15 minutes. PI label was added for 5 minutes before using the machine, with $200 \mu \mathrm{L}$ of $1 \times$ Binding buffer.

\section{Western blotting}

The cells were dissolved in RIPA lysis buffer and PMSF, and the protein concentration was measured with an ND2000 micro-spectrophotometer. Proteins were separated by SDS-PAGE ( $8 \%$ or $15 \%$ gels), transferred to PVDF membranes, and incubated with the primary antibody overnight. The next day, the membranes were incubated with the corresponding secondary antibody at room temperature for 2 hours. ECL chemiluminescent fluid and imaging system were used for exposure and data analysis. Primary antibody used: ApoM(China, ABclonal/A5336 1:1000), Caspase 3(UK, abcam/ab13847 1:1000),
Caspase 9(UK, abcam/ab69514 1:1000), Bax(US, CST/2772S 1:1000), Bcl 2(US, CST/3498S 1:1000), MMP-2 (China, BTL/BTL2798T 1:1000), $\beta$-actin(US, Sigma/A1978 1:5000) related protein expression levels.

\section{Transwell migration and invasion experiments}

Cells of each group were cultured to the logarithmic growth phase. After digestion and centrifugation, the cells were resuspend them in serum-free medium. We took $200 \mu \mathrm{L}$ of serum-free cell suspension $\left(1 \times 10^{5}\right.$ cells) of each group into the upper chamber of Transwell chamber, added $600 \mu \mathrm{L}$ of DMEM medium containing 20\% FBS to the lower chamber, and incubated it in a $5 \% \mathrm{CO}_{2}$ cell incubator. After incubation at $37{ }^{\circ} \mathrm{C}$ for $24 \mathrm{~h}$, the culture plate was taken out, washed with PBS once, fixed with $4 \%$ paraformaldehyde for 30 minutes, then discarded the solution, washed again. The migrated cells were stained with $1 \%$ crystal violet solution for 30 minutes. Under a fluorescent inverted microscope, images were captured in 5 fields randomly to count the number of cells penetrating the membrane randomly. The Matrigel glue needed to be taken out from $-20^{\circ} \mathrm{C}$ in a refrigerator and put at $4{ }^{\circ} \mathrm{C}$ overnight, The next day, we diluted the Matrigel glue with serum-free cell culture medium to $200 \mu \mathrm{L} / \mathrm{ml}$ at $4{ }^{\circ} \mathrm{C}$, added $50 \mu \mathrm{L}$ Matrigel on the Transwell polycarbonate membrane, and place the culture plate in the $37{ }^{\circ} \mathrm{C} 5 \% \mathrm{CO}_{2}$ cell incubator. After 2 hours, a basement membrane structure was formed. Then we added $600 \mu \mathrm{L}$ of $20 \%$ FBS DMEM culture medium to the basolateral side and $200 \mu \mathrm{L}$ of serum-free culture medium to the inside. The remaining steps were the same as the migration experiment.

\section{Tumor formation experiment in nude mice}

The mouse liver cancer Hepa1-6-control and Hepa1-6-ApoM-/- cells cultured to the logarithmic growth phase were adjusted to $1 \times 10^{7} / \mathrm{ml}$. The cells were injected into the left armpit of a 5-week-old BALB/c nude mouse (Nanjing Jicui Yaokang Biotechnology Co). The tumor diameter of the nude mouse is measured every 4 days. After 20 days, we sacrificed the mouse by cervical dislocation and taken the tumor in $-80^{\circ} \mathrm{C}$ for follow-up tests.

\section{Induction of liver cancer experiment in mice}

We weekly used N-nitrosodiethylamine solution (Shanghai Macleans Biochemical $35 \mathrm{mg} / \mathrm{kg}$ ) intraperitoneal injection of randomly selected 8-week-old healthy $30 \mathrm{C} 57 \mathrm{BL} / 6 \mathrm{~J}$ male WT and 30 ApoM $\%$ mice (Shanghai Southern Model Biology Research Center) and after injection At 0, 4, 8, 12, 16 and 20 weeks, 3 mice in each group were randomly selected to be anesthetized with $10 \%$ urethane. After 
weighing, blood was taken from the inner canthus for liver function test, the abdominal cavity was opened, and the whole liver was taken out. After recording, we stored it at $-80^{\circ} \mathrm{C}$ for subsequent testing.

\section{Statistical analysis}

GraphPad Prism 8.0 statistical software was used for data analysis. The experiments were repeated three times. The experimental data were expressed as mean \pm standard deviation $(\bar{x} \pm s)$. The t-test was used to compare the data between the two groups. $P<0.05$ was considered statistically significant.

\section{Results}

\section{The expression level of ApoM in cancer tissues is lower than that in adjacent tissues}

To initially verify whether there is a connection between ApoM and primary liver cancer, 50 matched samples of primary liver cancer tissues were selected from the TCGA database and analyzed. The expression level of the ApoM gene in the cancer tissues was lower than that in paracarcinoma tissues (Fig. 1A). another 23 clinical specimens of liver cancer were collected for verification, which was consistent with the results of bioinformatics analysis (Fig. 1B); at the same time, Compared with the normal mouse liver cell line AML12, Hepa1-6 showed a lower ApoM expression level (Fig. 1C).

Table 1. Clinicopathological analysis of cancer tissues and adjacent tissues in 23 patients with liver cancer (SD)

\begin{tabular}{llll}
\hline & Paracancerous & Carcinoma & $P$ value \\
\hline Age & & & \\
$\geq 50$ & $99.34(13.25)$ & $91.25(12.79)$ & 0.052 \\
$<50$ & $110.88(11.04)$ & $92.46(4.62)$ & 0.010 \\
Gender & & & \\
Male & $102.90(13.05)$ & $91.97(12.75)$ & 0.012 \\
Female & $97.24(15.54)$ & $89.68(4.76)$ & 0.241 \\
Tumor size & & & \\
Y & $101.27(17.67)$ & $92.39(11.69)$ & 0.020 \\
N & $105.68(15.54)$ & $83.81(7.07)$ & 0.071 \\
Necrosis & & & \\
Y & $106.29(12.81)$ & $90.27(13.30)$ & 0.024 \\
N & $99.32(13.56)$ & $92.14(10.80)$ & 0.085 \\
Sclerosis & & & \\
Y & $101.47(12.16)$ & $92.08(10.21)$ & 0.028 \\
N & $101.96(15.08)$ & $90.95(12.96)$ & 0.065 \\
Levels of differentiation & & & \\
Low & $108.54(11.65)$ & $87.51(8.89)$ & 0.001 \\
Middle & $97.80(13.98)$ & $96.56(14.03)$ & 0.811 \\
High & $98.44(13.37)$ & $90.93(10.73)$ & 0.245 \\
\hline
\end{tabular}
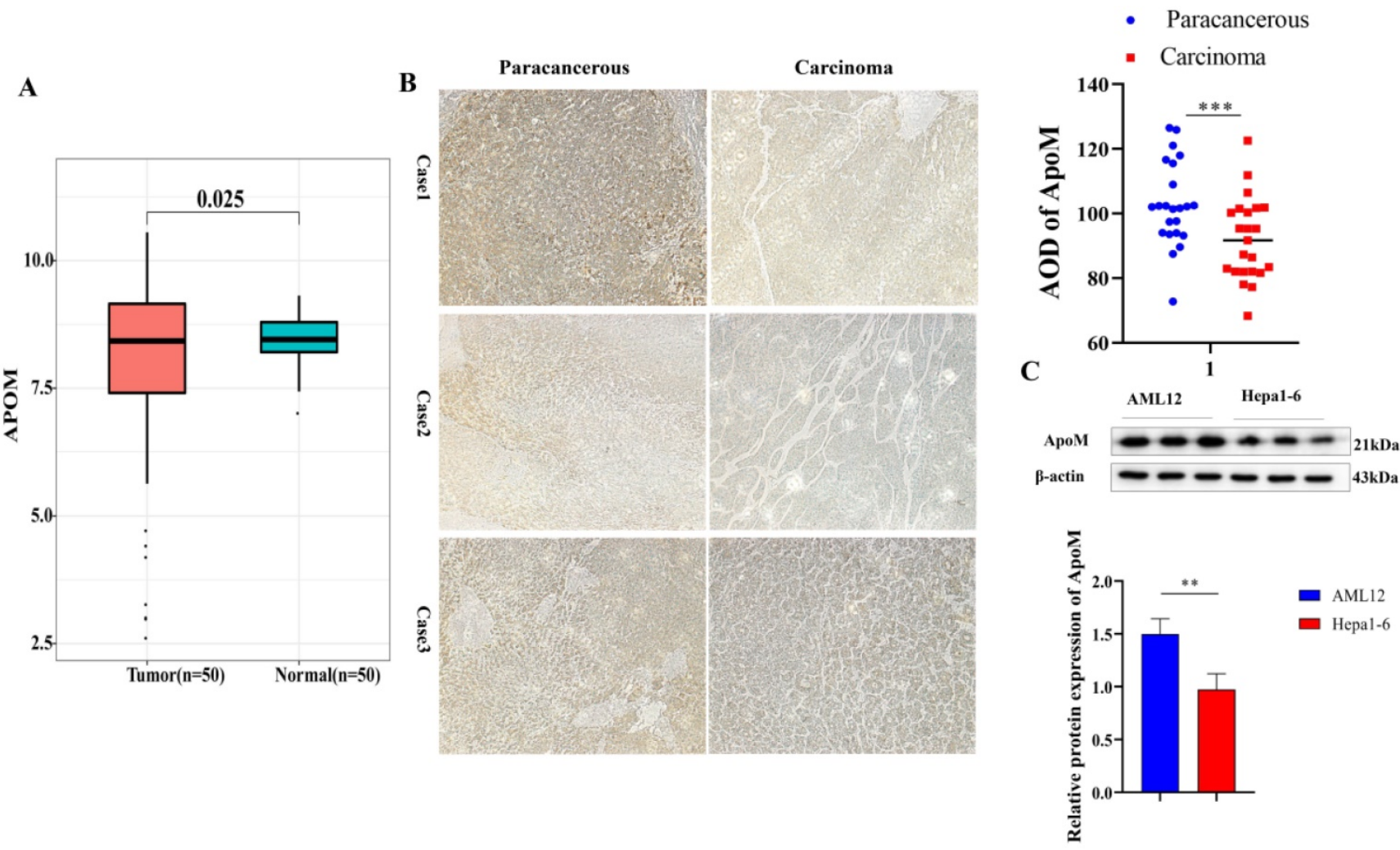

Figure 1. The expression level of ApoM in cancer tissues is lower than that in adjacent tissues. (A) 50 matched samples of primary liver cancer were selected from the TCGA database. The analysis showed that the expression level of ApoM gene in cancer tissues was lower than that in paracarcinoma tissues, and the difference was statistically significant $(P=0.025)$. (B) Using immunohistochemical method to detect the expression level of ApoM in cancer tissues and adjacent tissues of liver cancer patients, the expression level of ApoM in cancer tissues decreased significantly $(P=0.0063)$. (C) The expression level of ApoM in the normal mouse liver cell line $A M L 12$ was higher than that in the mouse liver cancer cell line Hepal-6 $(P=0.012) * p<0.05$, $* * p<0.03$, *** $p<0.01$. 


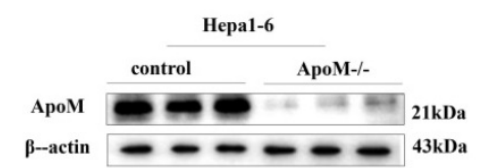

C

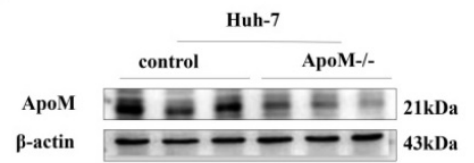

E

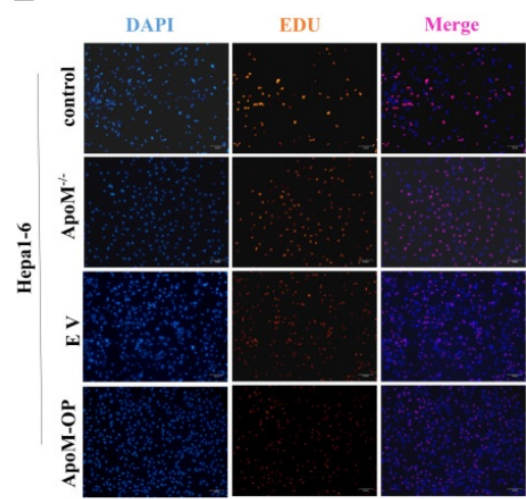

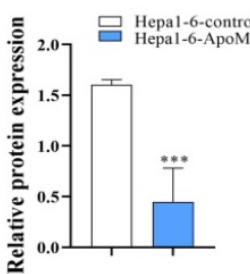

$\mathbf{B}$

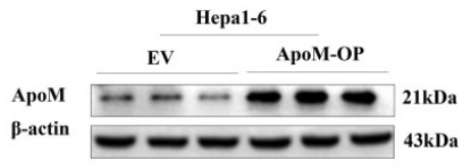

D
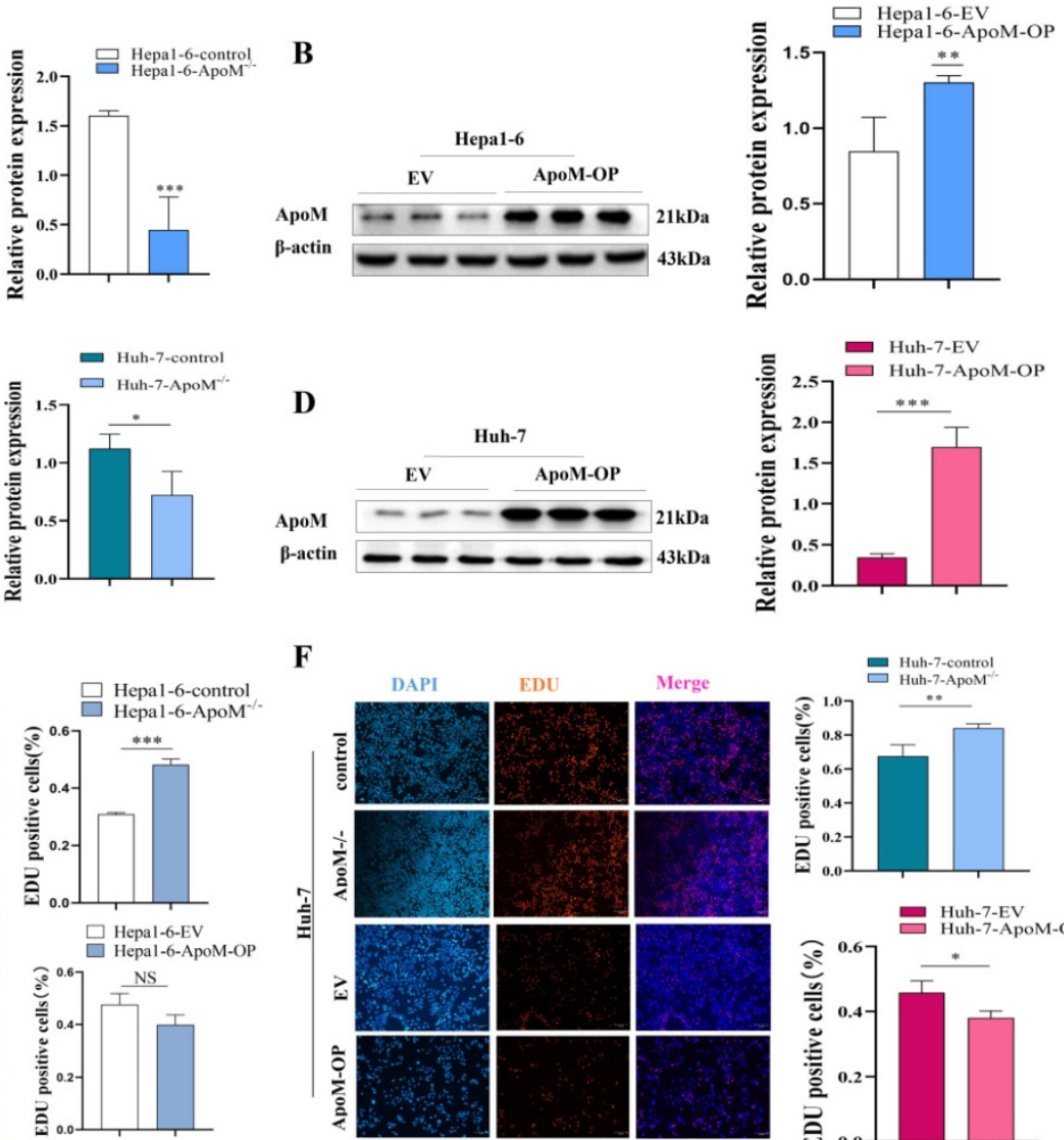

F

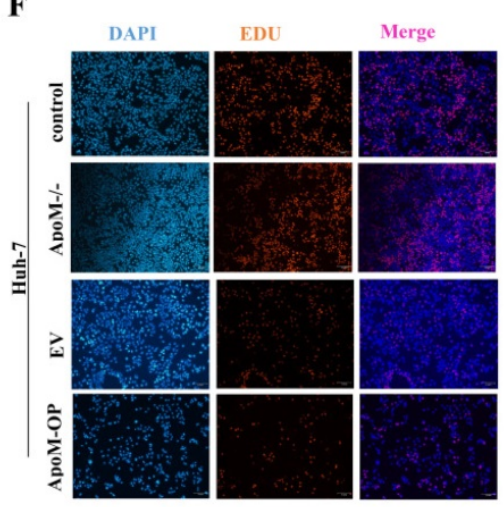

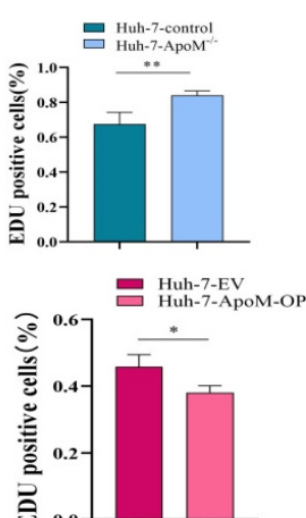

Figure 2. Expression level of the $A$ poM gene affects the proliferation of liver cancer cells. (A,B) Western blot method verified the knockout of the $A$ poM gene in the Hepal-6 cell line $(P=0.00049)$ and the overexpression model $(P=0.015)$ were successfully constructed. (C,D) Western blot method verified the knockout of the ApoM gene in the Huh-7 cell line $(P=0.044)$ and the overexpression model $(P=0.0000070)$ were successfully constructed. (E) Edu proliferation experiment showed that the cell proliferation activity of $A p O M$ gene deletion group increased $(P=0.00050)$, and $A p o M$ gene was overexpressed the cell proliferation activity had a downward trend $(P=0.070)$. $(F)$ The Edu proliferation experiment showed that the cell proliferation activity of the $A$ poM gene deletion group increased $(P=0.017)$, while the cell proliferation activity of the $A$ poM gene overexpression showed a downward trend in Huh-7 cells $(P=0.032) . \bar{x} \pm s, n \geq 3, *_{p}<0.05, *^{*} p<0.03, *^{*} p<0.01$.

\section{Expression level of the ApoM gene affects the proliferation of liver cancer cells}

The level of proliferation activity is one of the evaluation indicators of cell growth status. We constructed a mouse liver cancer cell model with ApoM gene deletion and overexpression and verified it (Fig. 2A, 2B). Edu experiments were used to determine whether the expression level of the ApoM gene affects the proliferation of liver cancer cells. The results showed that, compared with the control, the deletion of the ApoM caused an increase in cell proliferation rate, while it was decreased in overexpression of ApoM (Fig. 2E). In addition, consistent results were obtained in the human liver cancer cell line Huh-7. The deletion of ApoM gene promotes its proliferation, while the overexpression of ApoM gene inhibits proliferation (Fig. 2C, 2D, 2F).

\section{ApoM gene inhibits tumor formation and the liver cancer cell cycle}

In order to verify the in vitro experiment, we carried out in vivo experiments investigating tumor formation in BALB/c nude mice. Significantly different from the control group, the ApoM gene deletion group had a faster tumor growth rate (Fig. $3 \mathrm{~A})$, and we found that the difference between the two appeared on the 16th day after subcutaneous injection (Fig. 3C). The tumor tissue was removed to verify the expression level of ApoM protein (Fig. 3B), and we thus determined that the expression level of ApoM affected the proliferation of liver cancer cells. The results of cell cycle experiments suggested that, in liver cancer cells with ApoM gene deletion, the number of cells in the G0/G1 phase was reduced, and the proportion of cells in the $\mathrm{S}+\mathrm{G} 2 / \mathrm{M}$ phase was higher; in contrast, upon overexpression of $A p o M$, the G0/G1 phase was prolonged. Hence the decrease in the proliferation activity of the cells in the overexpression group was probably caused by blockage of the G0/G1 phase (Fig. 3D). Similarly, the cell cycle test of Huh-7-ApoM-\% model and Huh-7-ApoM-OP model is consistent with the above conclusions (Fig. 3E). 
A

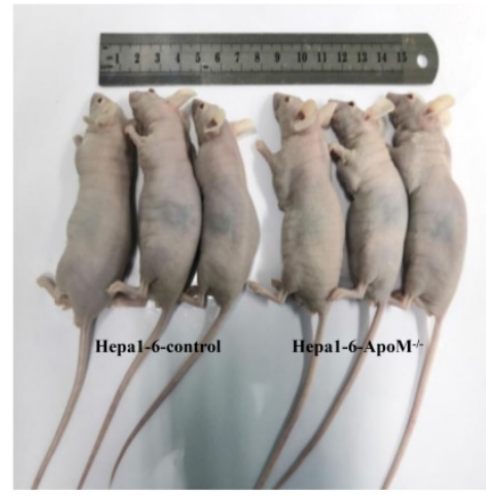

B

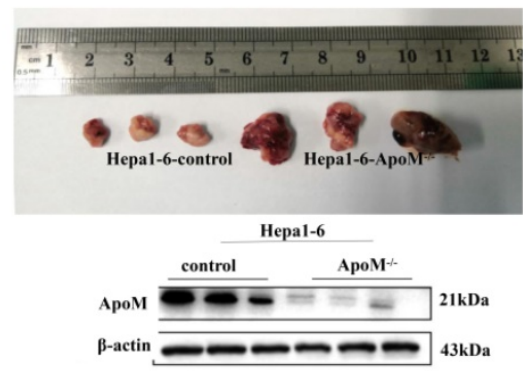

C

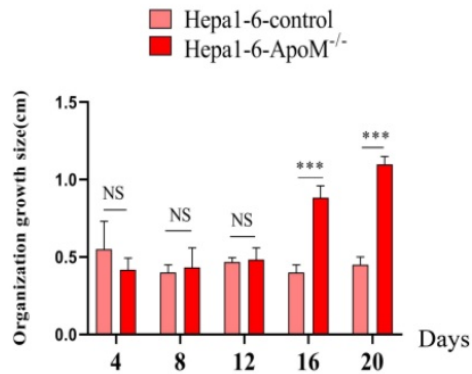

D

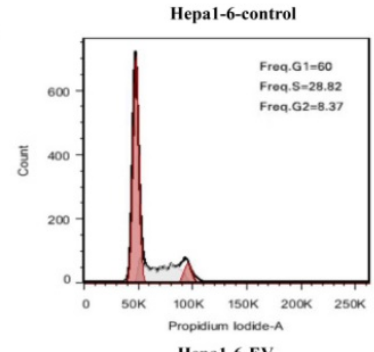

Hepa1-6-EV

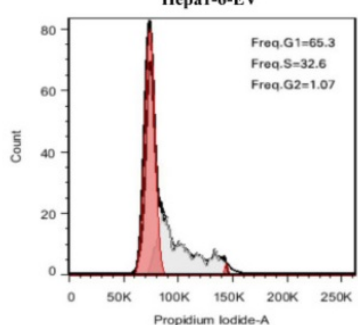

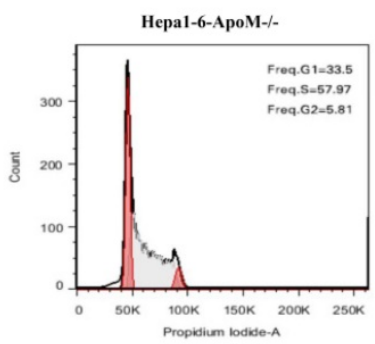

Hepa 1-6-ApoM-OP

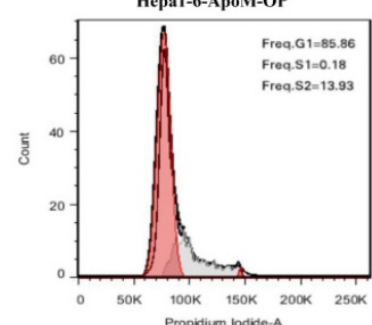

$\mathbf{E}$
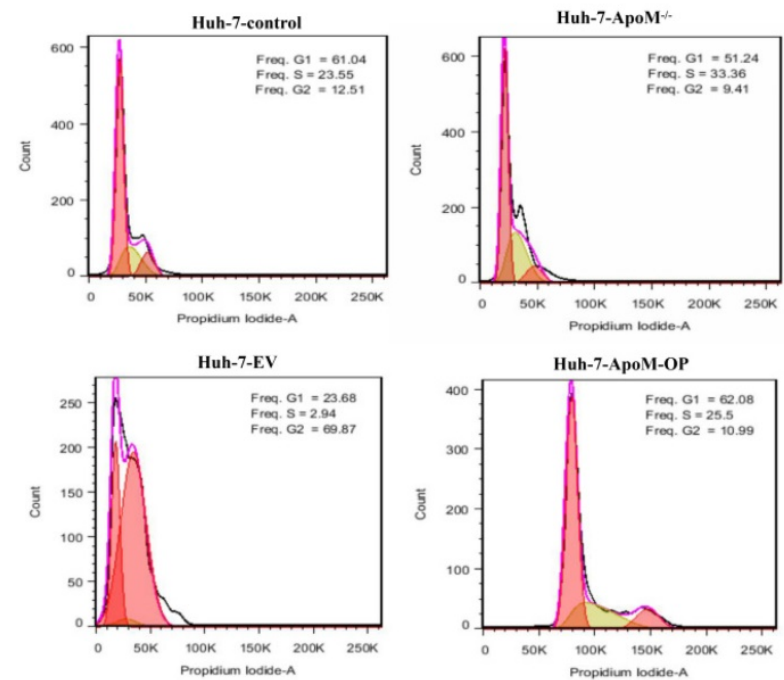

Figure 3. ApoM gene inhibits tumor formation rate and liver cancer cell cycle in nude mice. (A)The tumor formation experiment in nude mice showed that the same number of cells were injected subcutaneously in the two groups. Compared with the tumors in the Hepal-6-control group, the tumors grew faster in Hepal-6-ApoM- - . (B)The $A$ poM $\mathrm{M}^{-1}$ model in nude mouse tumors was successfully constructed by Western blot analysis. (C)The tumor growth size of nude mice showed that in the sixteenth day, the tumor in the Hepal-6-ApoM-- group was larger than that in the Hepal-6-control group $(P=0.00078)$. (D) Using cell cycle experiments to analyze the ApoM gene deletion group, the sum of G0/G2 and S phase is larger, and the proliferation rate is faster; the ApoM gene overexpression group, the G0/G1 phase is blocked and the proliferation rate is slower. (E) Similarly, the cell cycle test of Huh-7-ApoM-- model and Huh-7-ApoM-OP model is consistent with the above conclusions.

\section{ApoM gene promotes the apoptosis of liver cancer cells}

Flow cytometry analysis showed that, in the Hepa1-6-control group, the proportions of early apoptotic cells and late apoptotic cells were $23.3 \%$ and $1.01 \%$, respectively, at $12 \mathrm{~h}$ after transfection, $25.50 \%$ and $0.56 \%$, respectively, at $24 \mathrm{~h}$ after transfection, and $26.80 \%$ and $0.33 \%$, respectively, at $36 \mathrm{~h}$ after transfection. In the ApoM gene deletion group, the proportions of early apoptotic cells and late apoptotic cells were $8.79 \%$ and $5.50 \%$, respectively, $12 \mathrm{~h}$ after transfection, $10.40 \%$ and $4.84 \%$, respectively, $24 \mathrm{~h}$ after transfection, and $10.5 \%$ and $5.81 \%$, respectively, $36 \mathrm{~h}$ after transfection. Therefore, the ApoM gene deletion group had a lower level of apoptosis than the control group (Fig. 4A). In contrast, in the Hepa1-6-EV group, the proportions of early apoptotic cells and late apoptotic cells were $9.12 \%$ and $1.20 \%$, respectively, at $12 \mathrm{~h}$ after transfection, $13.70 \%$ and $0.78 \%$, respectively, at $24 \mathrm{~h}$ after transfection, and $13.40 \%$ and $6.23 \%$, respectively, at $36 \mathrm{~h}$ after transfection. In the Hepa1-6-ApoM-OP group, the proportions of early apoptotic cells and late apoptotic cells were $14.40 \%$ and $13.60 \%$, respectively, at $12 \mathrm{~h}$ after transfection, $13.60 \%$ and $18.30 \%$, respectively, at $24 \mathrm{~h}$ after transfection, and $38.00 \%$ and $0.69 \%$, respectively, at 36 $\mathrm{h}$ after transfection. These results indicated that the level of apoptosis increased after overexpression of ApoM (Fig. 4A).

\section{ApoM gene promotes the expression of key apoptotic proteins}

According to the flow cytometry results, we investigated further down to the molecular level. In the ApoM gene deletion group, the expression levels of Cleaved-caspase-3, Cleaved-caspase- 9 and Bax/Bcl-2 apoptosis-related proteins decreased. In the ApoM overexpression group, the expression of Cleaved-caspase-3, Cleaved-caspase- 9 and Bax/Bcl-2 apoptosis-related proteins increased (Fig. 5A, 5B). 
A

Hepa1-6-control
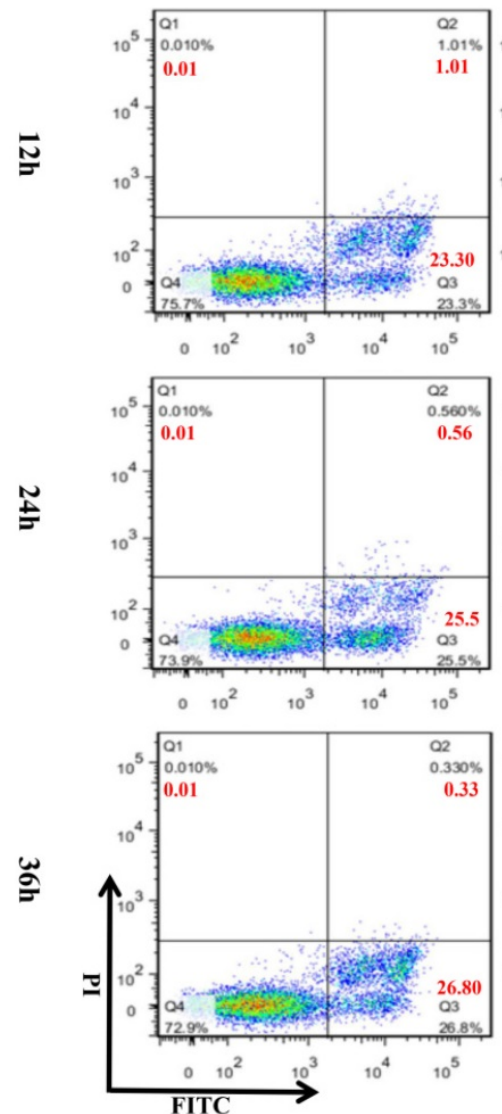

Hepa1-6-АpoM-/-
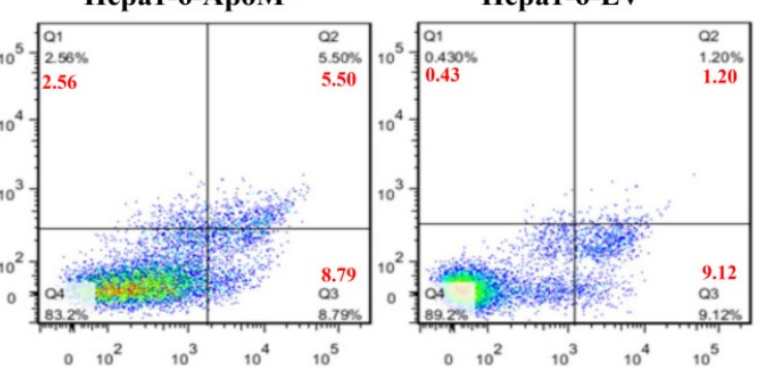

Hepa1-6-ApoM-OP
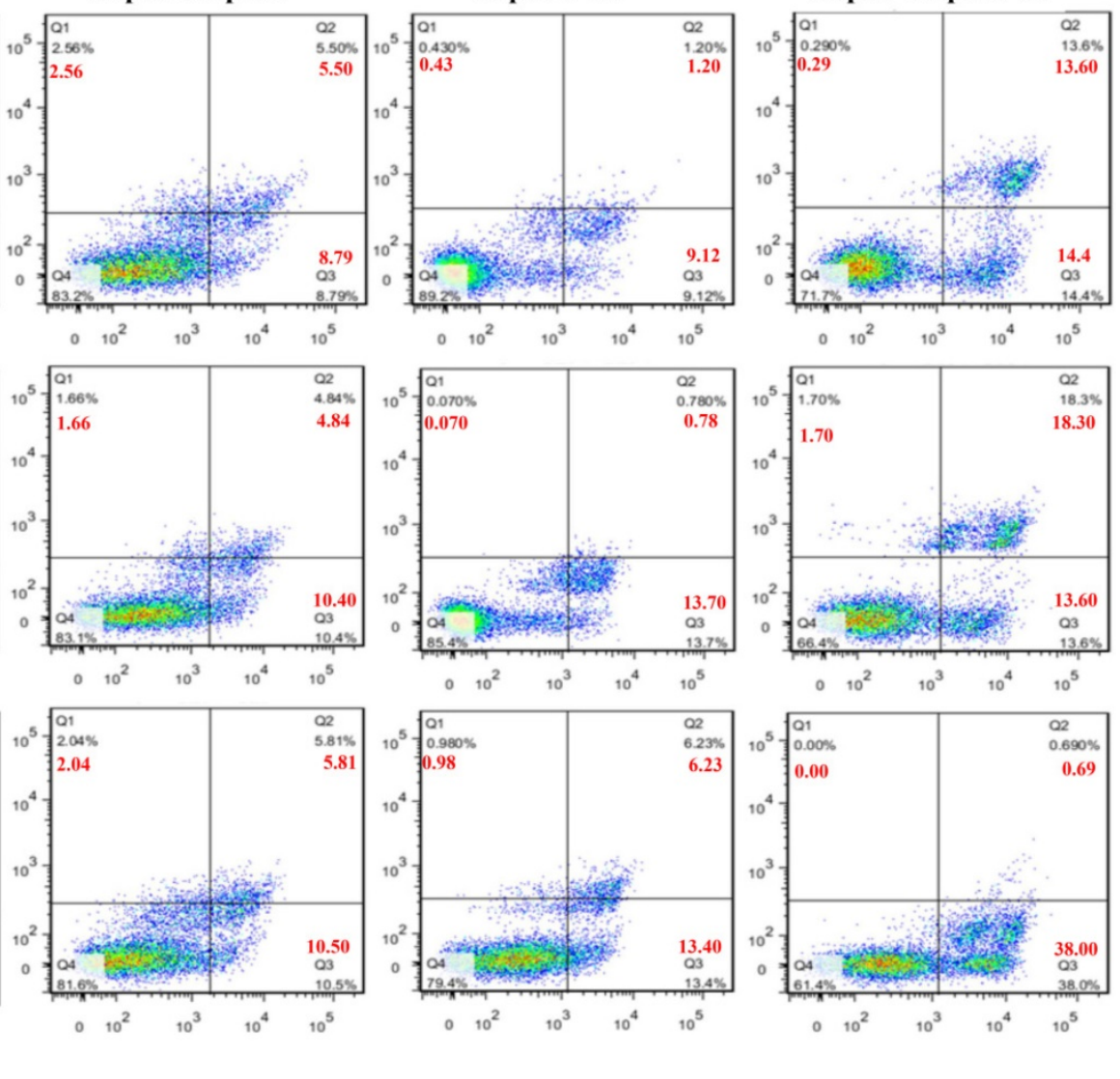

Figure 4. ApoM gene promotes apoptosis of liver cancer cells. (A) Flow cytometry analysis showed that the apoptotic level of cells transfected with ApoM-l- lentivirus changed after 12h, 24h and 36h. In Hepa 1-6 cells, the apoptosis rate of the ApoM gene deletion group was lower than that of the control group; while the cells transfected with ApoM overexpressing lentivirus were 12 hours, 24 hours and 36 hours later, the cells of the Hepal-6-ApoM-OP group The apoptosis rate was higher than that of the EV group.

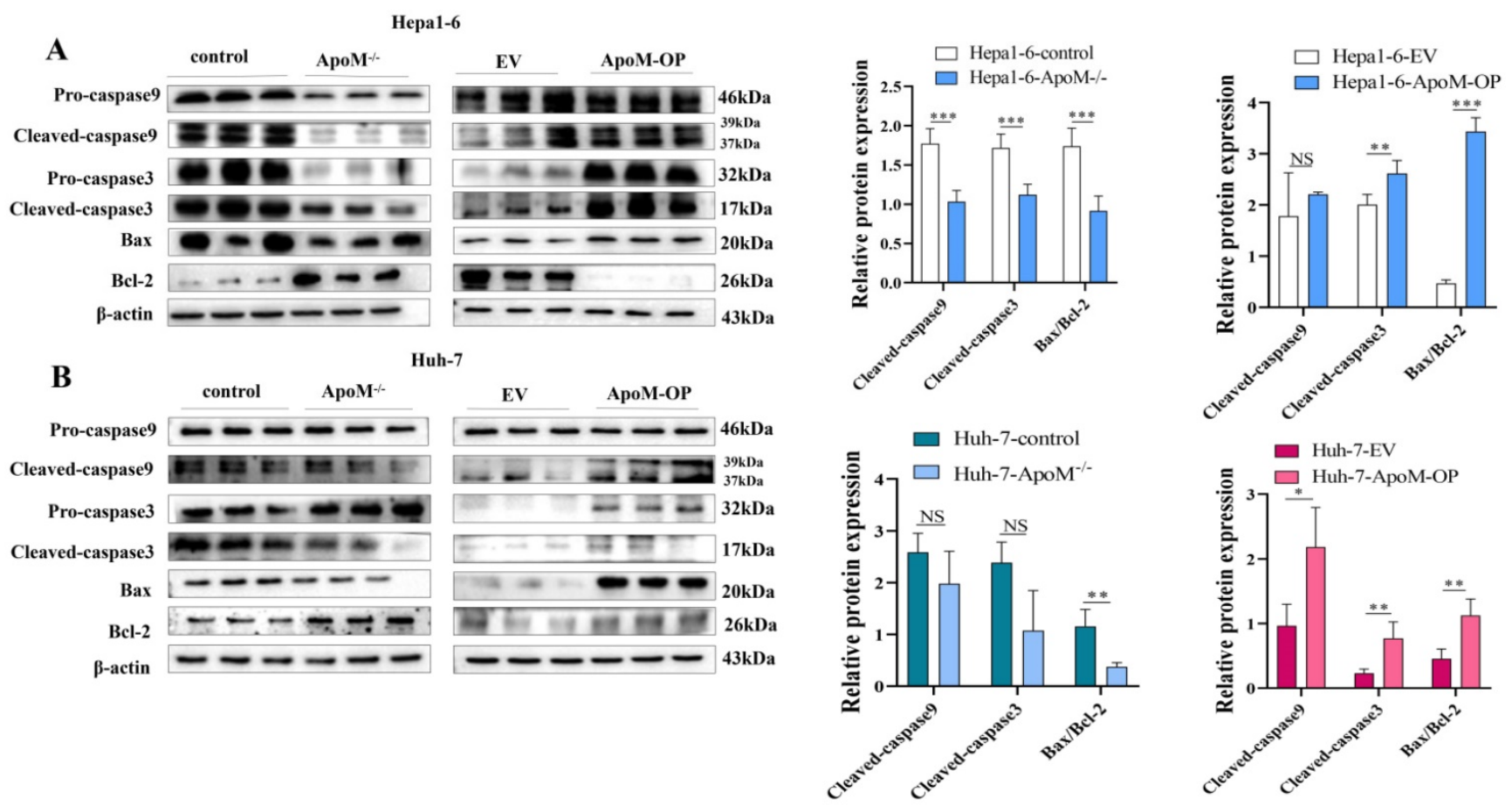

Figure 5. ApoM gene promotes the expression of key apoptotic proteins. (A, B) Western blot analysis of the expression levels of key apoptotic proteins, the results showed that the deletion of ApoM gene reduced the expression levels of Cleaved-caspase-3, Cleaved-caspase- 9 and Bax/Bcl-2 cell apoptosis-related proteins. The overexpression of $A$ poM gene increased the expression of Cleaved-caspase-3, Cleaved-caspase- 9 and Bax/Bcl- 2 apoptosis-related proteins, and statistical analysis was performed. $* p<0.05$, $* * p<0.03, * * * p<0.01$. 

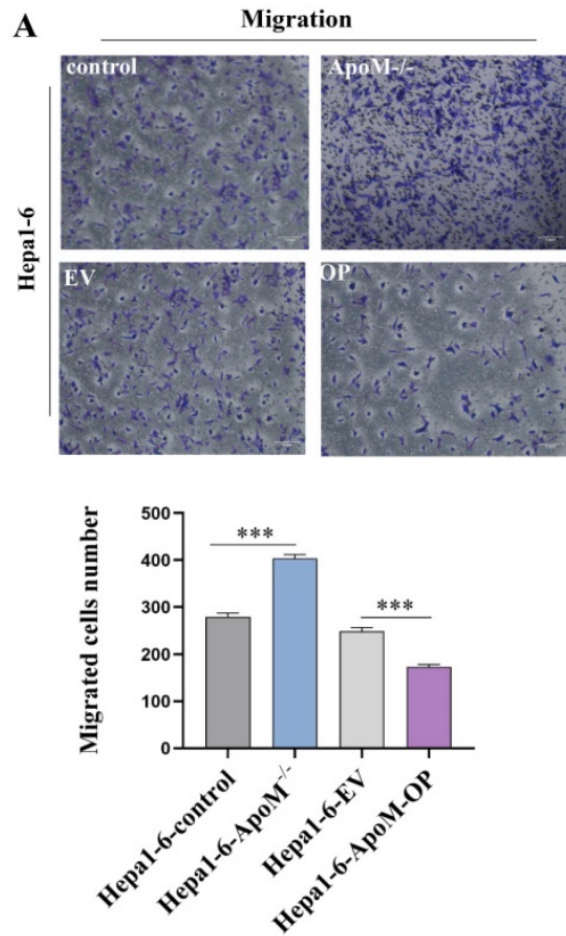

B
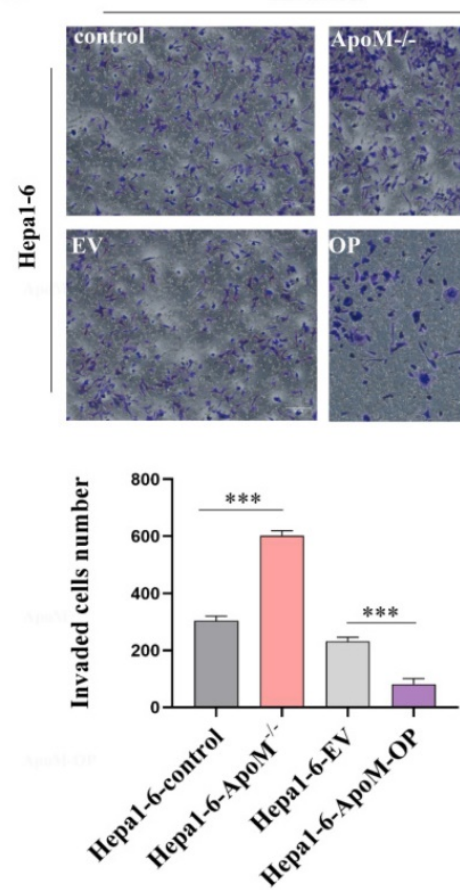

C
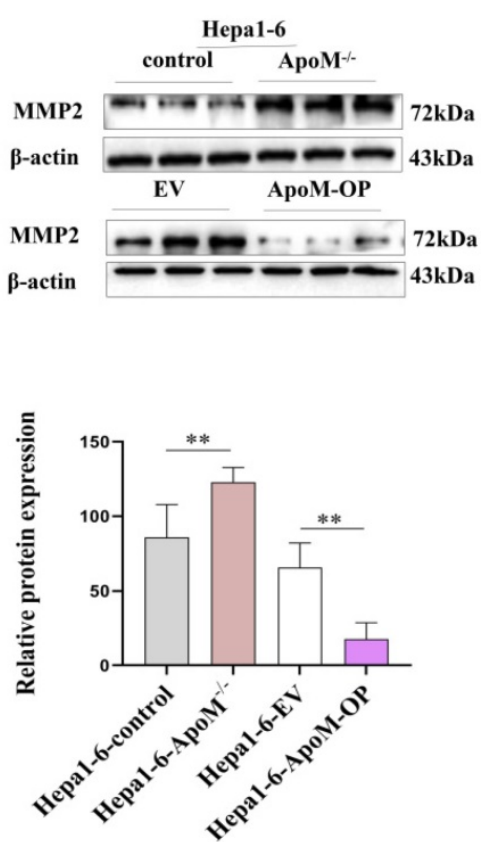

Figure 6. The deletion of ApoM gene aggravate the migration and invasion of Hepal-6 liver cancer cells. (A) The Transwell migration experiment indicated that the ApoM gene deletion group had stronger migration ability than the control group $(P=0.000044)$, while the overexpression group decreased $(P=0.00018)$. (B) The Transwell invasion experiment indicated that the $A$ poM gene deletion group had stronger invasion ability $(P=0.000025)$, while the overexpression group decreased $(P=0.00042)$. ( $C)$

Western blot analysis was used to analyze that the $A$ poM gene deletion group had a higher MMP- 2 protein expression level ( $P=0.027)$, and the overexpression group was the opposite $(P=0.027)$. All data were $\bar{x} \pm s$ of 3 independent measurements, $n \geq 3, * p<0.05, * * p<0.03, * * * p<0.01$.

\section{The deletion of ApoM gene aggravate the migration and invasion in liver cancer cells}

Studies have found that many patients with primary liver cancer die of liver cell metastasis. Transwell experiments were used to detect the migration and invasion abilities of cells in each group. The results suggested that the ApoM gene deletion group had stronger migration and invasion capabilities than the control group, while the overexpression group had decreased migration and invasion capabilities (Fig. 6A, 6B). At the same time, Western blot results showed that, compared to the control group, the ApoM gene deletion group had a higher level of MMP-2 protein expression (Fig. 6C) whereas the overexpression group had a lower level (Fig. 6C), suggesting that the ApoM gene inhibited the migration and invasion of liver cancer cells.

\section{Deletion of the ApoM gene accelerates the rate of liver cancer formation in mice treated of $\mathbf{N}$-nitrosodiethylamine induction}

WT and ApoM gene-deficient mice were intraperitoneally injected with $35 \mathrm{mg} / \mathrm{kg}$ $\mathrm{N}$-nitrosodiethylamine solution to induce liver cancer models. At the end of $0,4,8,12,16$, and 20 weeks, the mice were killed and the livers were taken to determine the ApoM gene pair and the effect of tumor formation rate in C57BL/6J mice. Compared with WT mice, ApoM gene-deficient mice developed liver tumors earlier (Fig. 7A, 7H). Throughout the induction process, At 12 weeks, the liver wet weight of the ApoM gene-deficient group was higher than that of the WT group and was statistically significant (Fig. 7C); from the $12^{\text {th }}$ week to the $16^{\text {th }}$ week, the weight of the ApoM gene-deficient mice decreased significantly (Fig. 7D). The expression level of ApoM in cancer tissues of WT mice was significantly lower than that of paracarcinoma tissues (Fig. 7B). Liver function ALT and AST levels of liver cancer induction model ApoM-- group were higher than those of WT group (Fig. 7E, 7F). In addition, the level of apoptosis in cancer tissues of WT mice was higher than that of Apo $\mathrm{M}^{-}$- mouse cancer tissues (Fig. 7G). Finally, the results of the survival curve indicated that the survival time of $A p o M^{-}-$mice was shorter than that of WT mice (Fig. 7I).

\section{Discussion}

To date, many apolipoproteins have been listed as tumor detection indicators. For example, some previously discovered apolipoproteins such as ApoB, ApoA1, and ApoE have been included in the biochemical tests of liver function and blood lipids[19, 20]. Compared with these apolipoproteins, ApoM was discovered late. Considering its distribution across 
various tissues in mice and humans, the expression level of the ApoM in the liver is by far the highest. As one of the pivotal organs of metabolism, the liver is vital in maintaining the steady state of glucose and lipid metabolism. As mentioned above, the occurrence and development of liver cancer are inseparable from abnormal lipid metabolism [21, 22]. In the future, non-alcoholic fatty liver disease will even become the main cause of liver cancer [23, 24]. And our previous studies have shown that the decline of $A p o M$ gene expression level can promote the accumulation of liver lipids by inhibiting the autophagy level of hepatocytes [8]. Therefore, will the down-regulation of the $A p o M$ gene disrupt the balance of liver metabolism and function? Will it participate in the occurrence and development of liver cancer? At least so far, the evidence is insufficient.

Here, we first prove whether ApoM is associated with primary liver cancer. Analyzing 50 matched samples of primary liver cancer in the TCGA database, the expression level of ApoM in cancer tissues was lower than that in paracarcinoma tissues (Fig. 1A). Use the collected 23 cases of primary liver cancer paired samples for verification (Fig. 1B). The expression of ApoM in cancer tissues and adjacent tissues is related to age $(<50, P=0.010)$, gender (male, $P=0.012)$, tumor size $(P=0.020)$, necrosis $(P=0.024)$ and liver cirrhosis $(P=0.028)$, factors related to poor differentiation $(P=0.0010)$ (Table 1$)$. The results are consistent with the database analysis results. The expression level of ApoM in the mouse liver cancer cell line was significantly lower than that in the normal liver cell line AML12 (Fig. 1C). Finally, in the liver cancer mouse model, the expression level of ApoM in the cancer tissue was also significantly lower than that in the paracarcinoma tissues (Fig. 7B). These evidences make us believe that ApoM may be a potential protective factor to inhibit the occurrence and development of primary liver cancer.
A

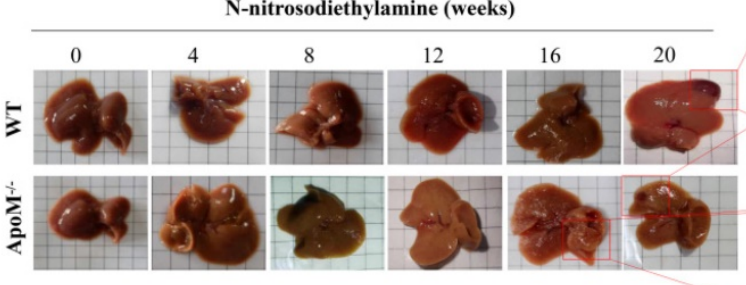

C

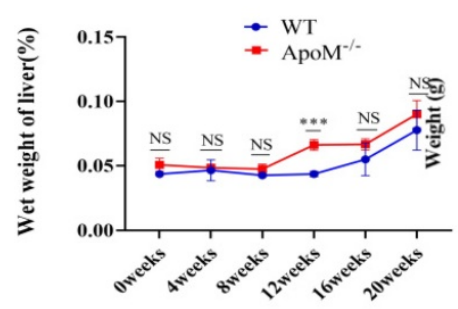

D

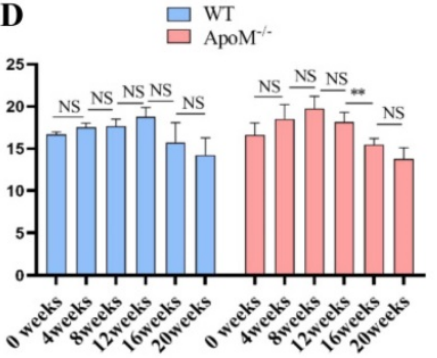

B

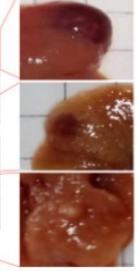

$\mathbf{E}$

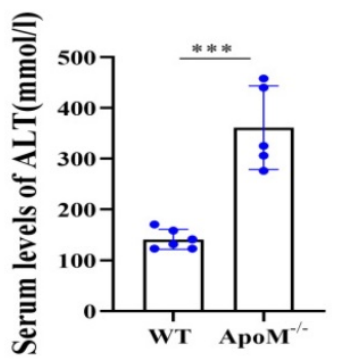

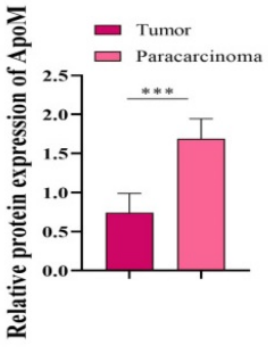

F

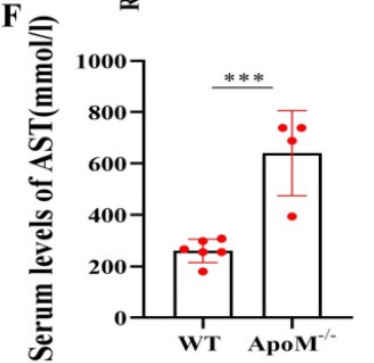

G

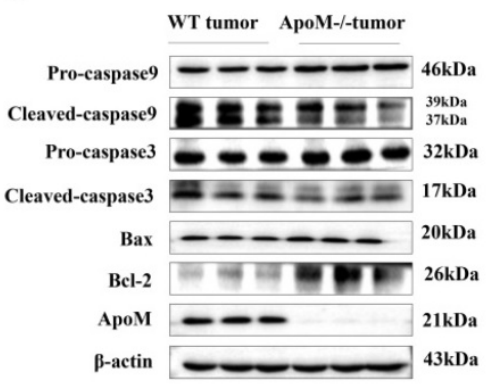

H

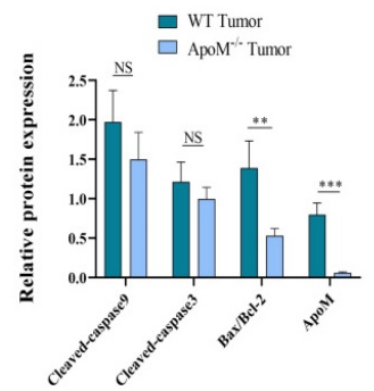

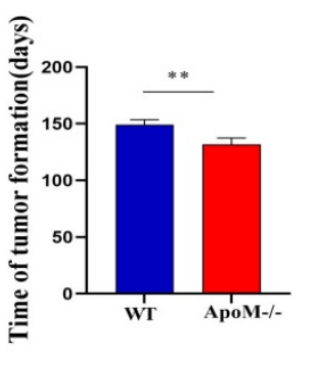

I

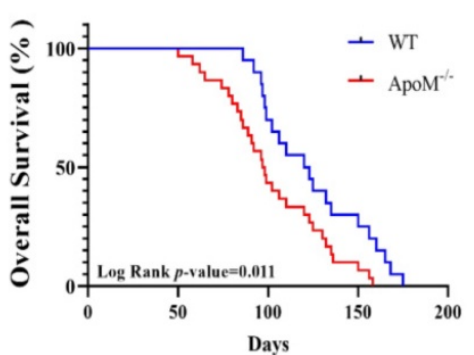

Figure 7. The deletion of $A$ poM gene accelerates the tumorigenesis rate of C57BL/6) mouse liver cancer induced by N-nitrosodiethylamine. (A) Livers were taken at 0, 4, 8, 12, 16 and 20 weeks. Compared with WT mice, ApoM gene-deficient mice developed tumors earlier under $\mathrm{N}$-nitrosodiethylamine induction. (B) Western blot assay showed that the ApoM level in the cancer tissue of WT mice in the liver cancer induction model group was lower than that in the paracarcinoma tissues (P=0.010). (C) Compared with the WT group, the ApoM gene deletion group had higher wet liver weight per month. At 12 weeks, the wet liver weight of the ApoM gene deletion group was higher than that of the WT group and was statistically significant $(P=0.0048)$. (D) The body weight of mice in the WT group increased from 0 weeks to 12 weeks, and their body weight decreased after 12 weeks. The body weight of mice in the ApoM gene deletion group increased from 0 to 8 weeks, and their body weight decreased after 8 weeks. (E, F) Liver function ALT $(P=0.00013)$ and AST $(P=0.00061)$ levels of liver cancer induction model $A p 0 \mathrm{M}^{--}$group are higher than those of WT group, and have statistical significance. (G) Western blot analysis of liver cancer induction model, the key apoptosis proteins Cleaved-caspase9 $(P=0.20)$, Cleaved-caspase3 $(P=0.26)$, Bax/Bcl-2 $(P=0.014)$ in wild mice were higher than $A p 0 M$ gene deletion group. $(P=0.0011)$. (H) Compared with the WT group, the $A p o M$ gene deletion group has a faster time for liver tumors, which was statistically significant $(P=0.028)$. (I) The survival of $A p o M^{-1-}$ mice was shorter than that of WT mice $(P=0.011)$. 
Unreserved rapid growth is one of the characteristics of tumor cells, and it is also the highlight of increased cell proliferation activity. Edu cell proliferation experiments showed that the proliferation activity in the knockout group increased significantly. Although it was not statistically significant, the proliferation activity in the overexpression group decreased (Fig. 2E, 2F). In order to further verify the experimental results at the ex vivo level, we subcutaneously injected the control group cells and the constructed knockout group cells into nude mice and observed the tumor formation rates of the two. We found that axillary lumps showed up faster in the knockout group of nude mice and were significantly larger than those in the control group of nude mice on the 16th day (Fig. 3A, 3C). Accordingly, we concluded that the deletion of the ApoM gene promotes the proliferation of the mouse liver cancer cells Hepa1-6. Cell cycle experiments further showed that the proportion of knockout group cells decreased in the G0/G1 phase, and the proportion in the $\mathrm{S}+\mathrm{G} 2 / \mathrm{M}$ phase increased, while the overexpression group was blocked in the G0/G1 phase (Fig. 3D, 3E).

Apoptosis is a programmed cell death mode regulated by genes. It is an effective way to eliminate senescence, damage, and precancerous cells in the human body [25], and down-regulation of apoptosis is one of the reasons for the excessive proliferation of tumor cells [26]. In order to explore whether the level of ApoM gene expression affects the level of apoptosis of liver cancer cells, we used flow cytometry to detect the level of apoptosis in the knockout group and overexpression group induced by cisplatin. Different sampling times were introduced to reduce errors caused by transfection efficiency and expression efficiency. The results showed that the knock-out group had a lower level of apoptosis than the control group, while the overexpression group displayed the opposite trend (Fig. 4A). In addition, the WB results further supported the above results. The knockout group down-regulated (and the over-expression group up-regulated) the level of activity in the mitochondrial apoptosis pathway (Fig. 5A, 5B).

The growth status of tumor cells is certainly important, but more ominous is the ability of tumor cells to spread from the original site to surrounding tissues and even to metastasize further away. From the perspective of liver cancer, about $60 \%$ of patients' exhibit metastasis at the time of treatment, and even throughout active treatment there will still be a high recurrence rate [27]. Inhibiting the migration and invasion of cancer cells is an important prerequisite for improving postoperative survival and ensuring a good prognosis. Unfortunately, we observed that knockout of the ApoM gene increased the migration and invasion capabilities of mouse liver cancer cells, while overexpression did not (Fig. 6).

In summary, ApoM plays a role in inhibiting the development of liver cancer cells as shown in proliferation, apoptosis, migration, and invasion related experiments. Does ApoM also play a role in the development of liver cancer? We used WT mice as the control group and ApoM knockout mice as the experimental group. We used N-nitrosodiethylamine to induce primary liver cancer, and observed whether the level of ApoM gene expression affected the occurrence of primary liver cancer. As expected, tumors appeared earlier in the livers of knockout mice (Fig. 7A, 7H). In addition, we also observed that when $\mathrm{N}$-nitrosodiethylamine was induced for 12 weeks, the wet weight of the liver of the knockout mice increased the most (Fig. 7C), while the weight of knockout mice decreased significantly after 12 weeks (Fig. 7D). We also found that the level of apoptosis in WT mice was higher than that in ApoM---mice (Fig. 7G). The survival time of $A p o M^{-/}$mice was shorter than that of WT mice (Fig. 7I).

Obviously, in both in vitro and in vivo experiments, ApoM plays a role in inhibiting the occurrence and development of primary liver cancer. Here, we have obtained the corresponding results by regulating the expression level of the ApoM gene. In view of the increasing importance of the liver lipid metabolism environment in malignant liver disease, we initially took the influence of ApoM on liver lipid metabolism as a clue and entry point to explore the correlation between ApoM and liver cancer. And we also found that the down-regulation of the expression level of $A p o M$ gene can lead to impaired liver function (Fig. 7E, 7F). The damaged liver will inevitably lead to a decrease in ApoM expression. Will this form a vicious circle? Therefore, in future work, we will further explore the specific mechanism that enables ApoM to affect the occurrence and development of liver cancer by regulating lipid metabolism. This will significantly improve our knowledge of the overall mechanism of primary liver cancer and enable us to find potential prevention and treatment targets.

\section{Acknowledgements}

This work was supported by Anhui Science and Technology Public Relations (Project No. 1804h08020241) and Anhui University Natural Science Research Project (Project Number: KJ2020A0612).

\section{Ethical approval and participation consent}

The experimental animal program involved has been approved by the Experimental Animal Welfare and Ethics Committee of Wannan Medical College. 
Clinical specimens have been approved by the Ethics Committee of Scientific Research and New Technology of Yijishan Hospital of Wannan Medical College.

\section{Author contribution}

Yaping Bai was responsible for most of the article experiments, analyzed the data in the existing literature, and wrote the article; Wenjun Pei and Xiao Zhang participated in the project design and revised the manuscript; Huihao Zheng participated in the coating of the ApoM gene lentivirus; Lisheng $\mathrm{Hu}$ and Jiao Min participated in the part Cell function experiments; Shuangqiu Du and Changchun Hua participated in some in vivo experimental studies; Gong Zuyue played a key role in later English polishing; Yao Zhang and Jialin Gao were responsible for overall control and guidance of the subject.

\section{Competing Interests}

The authors have declared that no competing interest exists.

\section{References}

1. Pascual S, Herrera I, Irurzun J. New advances in hepatocellular carcinoma. World J Hepatol. 2016; 8: 421-38.

2. Rawla P, Sunkara T, Muralidharan P, Raj JP. Update in global trends and aetiology of hepatocellular carcinoma. Contemp Oncol (Pozn). 2018; 22: $141-50$

3. Chen K, Ma J, Jia X, Ai W, Ma Z, Pan Q. Advancing the understanding of NAFLD to hepatocellular carcinoma development: From experimental models to humans. Biochim Biophys Acta Rev Cancer. 2019; 1871: 117-25.

4. Shi C, Xue W, Han B, Yang F, Yin Y, Hu C. Acetaminophen aggravates fat accumulation in NAFLD by inhibiting autophagy via the AMPK/mTOR pathway. Eur J Pharmacol. 2019; 850: 15-22.

5. Dou X, Li S, Hu L, Ding L, Ma Y, Ma W, et al. Glutathione disulfide sensitizes hepatocytes to TNFalpha-mediated cytotoxicity via IKK-beta S-glutathionylation: a potential mechanism underlying non-alcoholic fatty liver disease. Exp Mol Med. 2018; 50: 7.

6. Bosteen MH, Madsen Svarrer EM, Bisgaard LS, Martinussen T, Madsen M, Nielsen LB, et al. Effects of apolipoprotein $\mathrm{M}$ in uremic atherosclerosis. Atherosclerosis. 2017; 265: 93-101.

7. Ruiz M, Frej C, Holmer A, Guo LJ, Tran S, Dahlback B. High-Density Lipoprotein-Associated Apolipoprotein M Limits Endothelial Inflammation by Delivering Sphingosine-1-Phosphate to the Sphingosine-1-Phosphate Receptor 1. Arterioscler Thromb Vasc Biol. 2017; 37: 118-29.

8. Zhang X, Zhang P, Gao J, Huang Q. Autophagy dysregulation caused by ApoM deficiency plays an important role in liver lipid metabolic disorder. Biochem Biophys Res Commun. 2018; 495: 2643-48.

9. Hu YW, Chen ZP, Hu XM, Zhao JY, Huang JL, Ma X, et al. The miR-573/apoM/Bcl2A1-dependent signal transduction pathway is essential for hepatocyte apoptosis and hepatocarcinogenesis. Apoptosis. 2015; 20: 1321-37.

10. Katzke VA, Sookthai D, Johnson T, Kuhn T, Kaaks R. Blood lipids and lipoproteins in relation to incidence and mortality risks for CVD and cancer in the prospective EPIC-Heidelberg cohort. BMC Med. 2017; 15: 218

11. Yang G, Wang Y, Feng J, Liu Y, Wang T, Zhao M, et al. Aspirin suppresses the abnormal lipid metabolism in liver cancer cells via disrupting an NFkappaB-ACSL1 signaling. Biochem Biophys Res Commun. 2017; 486: $827-32$

12. Zhen N, Yang $Q$, Wu Q, Zhu X, Wang $Y$, Sun F, et al. A novelly synthesized phenanthroline derivative is a promising DNA-damaging anticancer agent inhibiting G1/S checkpoint transition and inducing cell apoptosis in cancer cells. Cancer Chemother Pharmacol. 2016; 77: 169-80.

13. Arbabi S, Maier RV. Mitogen-activated protein kinases. Crit Care Med. 2002; 30: S74-S79.

14. Kanda T, Matsuoka S, Yamazaki M, Shibata T, Nirei K, Takahashi H, et al. Apoptosis and non-alcoholic fatty liver diseases. World J Gastroenterol. 2018; 24: $2661-72$

15. Zhang $\mathrm{Z}$, Wang $\mathrm{X}, \mathrm{Wu} \mathrm{W}$, Wang J, Wang $\mathrm{Y}, \mathrm{Wu} \mathrm{X}$, et al. Effects of matrine on proliferation and apoptosis in gallbladder carcinoma cells (GBC-SD). Phytother Res. 2012; 26: 932-7.
16. Fulda S. Targeting apoptosis for anticancer therapy. Semin Cancer Biol. 2015; 31: 84-8.

17. Pastushenko I, Brisebarre A, Sifrim A, Fioramonti M, Revenco T, Boumahdi S, et al. Identification of the tumour transition states occurring during EMT. Nature. 2018; 556: 463-68

18. Luengo A, Gui DY, Vander Heiden MG. Targeting Metabolism for Cancer Therapy. Cell Chem Biol. 2017; 24: 1161-80.

19. Shah SS, Desai HG. Apolipoprotein deficiency and chronic liver disease. J Assoc Physicians India. 2001; 49: 274-8.

20. Katsuramaki T, Mizuguchi T, Kawamoto M, Yamaguchi K, Meguro M, Nagayama $M$, et al. Assessment of nutritional status and prediction of postoperative liver function from serum apolioprotein A-1 levels with hepatectomy. World J Surg. 2006; 30: 1886-91.

21. Ackerman D, Simon MC. Hypoxia, lipids, and cancer: surviving the harsh tumor microenvironment. Trends Cell Biol. 2014; 24: 472-8.

22. Pope ED, 3rd, Kimbrough EO, Vemireddy LP, Surapaneni PK, Copland JA, 3rd, Mody K. Aberrant lipid metabolism as a therapeutic target in liver cancer. Expert Opin Ther Targets. 2019; 23: 473-83.

23. Piscaglia F, Svegliati-Baroni G, Barchetti A, Pecorelli A, Marinelli S, Tiribelli C, et al. Clinical patterns of hepatocellular carcinoma in nonalcoholic fatty liver disease: A multicenter prospective study. Hepatology. 2016; 63: 827-38.

24. Kanwal F, Kramer JR, Duan Z, Yu X, White D, El-Serag HB. Trends in the Burden of Nonalcoholic Fatty Liver Disease in a United States Cohort of Veterans. Clin Gastroenterol Hepatol. 2016; 14: 301-8 e1-2.

25. Pistritto G, Trisciuoglio D, Ceci C, Garufi A, D'Orazi G. Apoptosis as anticancer mechanism: function and dysfunction of its modulators and targeted therapeutic strategies. Aging (Albany NY). 2016; 8: 603-19.

26. Mollereau B, Perez-Garijo A, Bergmann A, Miura M, Gerlitz O, Ryoo HD, et al. Compensatory proliferation and apoptosis-induced proliferation: a need for clarification. Cell Death Differ. 2013; 20: 181

27. Kornberg A, Witt U, Kornberg J, Friess H, Thrum K. Extended Ischemia Times Promote Risk of HCC Recurrence in Liver Transplant Patients. Dig Dis Sci. 2015; 60: 2832-9. 